\title{
$\mathrm{KJCCM}$
}

\section{Recombinant Activated Factor VII as a Second Line Treatment for Postpartum Hemorrhage}

\author{
Soon Chang Park', Seok Ran Yeom ${ }^{1,2}$, Sang Kyoon Han ${ }^{1}$, Young Mo Jo ${ }^{1}$, and Hyung Bin Kim ${ }^{1}$ \\ ${ }^{1}$ Department of Emergency Medicine, Pusan National University Hospital, Busan; ' Department of Emergency Medicine, Pusan National University School of Medicine, Yangsan, Korea
}

Background: Severe or massive postpartum hemorrhage (PPH) has remained a leading cause of maternal mortality for decades across the world and it results in critical obstetric complications. Recombinant activated factor VII (rFVIla) has emerged as a gold standard adjunctive hemostatic agent for the treatment of life-threatening PPH refractory to conventional therapies although it remains off-licensed for use in PPH. We studied the effects of rFVIla on coagulopathy, transfusion volume, prognosis, severity change in Korean PPH patients.

Methods: A retrospective review of medical records between December 2008 and March 2011 indicating use of rFVlla in severe PPH was performed. We compared age, rFVIla treatment, transfusion volume, and Sequential Organ Failure Assessment (SOFA) score at the time of arrival in the emergency department and after 24 hours for patients whose SOFA score was 8 points or higher.

Results: Fifteen women with SOFA score of 8 and above participated in this study and eight received $\mathrm{rFV}$ Ila administration whereas seven did not. Patients' mean age was $31.7 \pm 7.5$ years. There was no statistically significant difference in initial and post- 24 hours SOFA scores between patients administered rFVIla or not. The change in SOFA score between initial presentation and after 24 hours was significantly reduced after $r F V$ lla administration $(P=0.016)$.

Conclusions: This analysis aimed to support that the administration of rFVIla can reduce the severity of life-threatening PPH in patients. A rapid decision regarding the administration of $r F V I l a$ is needed for a more favorable outcome in severe PPH patients for whom there is no effective standard treatment.

Key Words: factor VIla; maternal death; organ dysfunction scores; postpartum hemorrhage; recombinant proteins.

\section{Introduction}

Despite major treatment advances over the past several decades, postpartum hemorrhage (PPH) continues to be a potentially life-threatening obstetric complication and a significant cause of maternal morbidity and mortality, and is also associated with severe medical conditions including hypovolemic shock, multi-organ failure, disseminated intravascular coagulation, acute renal failure, and acute respiratory distress syndrome. The World Health Organization found that PPH affected approximately $2 \%$ of all birthing women and accounted for $25 \%$ of all global maternal deaths (about 140,000 per year) in 2014 [1]. Statistics Korea [2] has reported that 0.1 patient per 100,000 births dies from massive hemorrhage, accounting for $11 \%$ of all maternal deaths in 2014 . Thus, in Korea, PPH remains a major complication in the peripartum period, and a common cause of maternal death.

Received on August 31, 2016 Revised on September 13, 2017 Accepted on October 9, 2017

Correspondence to: Seok Ran Yeom, Department of Emergency Medicine, Pusan National University Hospital, 179 Gudeok-ro, Seo-gu, Busan 49241, Korea Tel: +82-51-240-7503, Fax: +82-51-253-6472, E-mail: seokrany@pusan.ac.kr

cc This is an Open Access article distributed under the terms of the Creative Commons Attribution Non-Commercial License (http://creativecommons.org/ licenses/by-nc/4.0/) which permits unrestricted non-commercial use, distribution, and reproduction in any medium, provided the original work is properly cited. Copyright (c) 2017 The Korean Society of Critical Care Medicine 
Traditionally, PPH has been defined as a blood loss of $\geq 500 \mathrm{ml}$ during the peripartum period, and severe $\mathrm{PPH}$ as blood loss of $\geq 1,000 \mathrm{ml}$. PPH is subdivided into primary PPH (developing within 24 hours of delivery) and secondary PPH (developing between 24 hours and 6 weeks postpartum) [3]. PPH is medically managed using uterotonic agents (e.g., intravenous oxytocin and/or ergometrine, and sublingual misoprostol), and/or transfusion of isotonic crystalloids to replace blood loss. If patients do not respond to uterotonics, thus exhibiting uncontrolled bleeding, nonsurgical interventions including uterine massage, uterine balloon tamponade, uterine artery embolization, and/or surgical interventions, are recommended to treat $\mathrm{PPH}$ attributable to uterine atony. Hysterectomy is the treatment of last resort [4].

However, even after such varied treatments, PPH may still cause death or complex problems such as coagulopathy triggered by massive transfusions or amniotic fluidrelated issues. Mortality and morbidity associated with $\mathrm{PPH}$ remain high not only in developing countries but also in advanced countries.

Recombinant activated factor VII (rFVIIa; Novoseven, Novo Nordisk, Bagsvaerd, Denmark) is emerging as a novel therapy for the treatment of life- or fertility-threatening PPH unresponsive to standard therapy and may, in some cases, prevent the need for peripartum hysterectomy [5]. However, the World Health Organization considers there is insufficient evidence to strongly recommend use of the drug [4].

In 1998, the effectiveness of rFVIIa was recognized, and rFVIIa was approved for use to control bleeding in patients with hemophilia A or B who expressed inhibitors of coagulation factors. In recent years, hundreds of cases of off-label use of rFVIIa as a hemostatic agent to control severe/massive hemorrhage in a variety of clinical situations have been investigated and the value of rFVIIa has been documented. The outstanding efficacy of off-label rFVIIa in the management of life-threatening obstetric hemorrhage has been recognized by many clinicians, resulting in guidelines for the use of rFVIIa in the management of major obstetric hemorrhage [5]. However, no Korean study has yet evaluated the use of rFVIIa to treat PPH. Thus, we studied the effects of rFVIIa on coagulopathy, transfusion volume required, prognosis, and changes in the severity of PPH in Korean patients.

\section{Materials and Methods}

\section{1) Data collection}

We retrospectively reviewed the medical records of PPH patients admitted to Pusan National University Hospital from December 2008 to March 2011. We used the Sequential Organ Failure Assessment (SOFA) score to rate illness severity. Patients with SOFA scores $<7$ points were excluded, and those with scores $\geq 8$ points were selected for investigation. Also, patients who had suffered cardiac arrest before arrival at our hospital were excluded.

We reviewed the medical records for age, rFVIIa use, vital signs at the time of arrival at hospital, hemoglobin level, prothrombin time, activated prothrombin time, and the amount of blood components required. The $\triangle$ SOFA values (the differences between SOFA scores on 1-3 day of PPH evolution and SOFA scores on arrival) correlated with mortality in several prior studies. We also reviewed SOFA scores at the time of arrival at the hospital and 24 hours later.

\section{2) rFVIla administration}

We conducted this study in compliance with the principles of the Declaration of Helsinki. The protocol of this study was reviewed and approved by the Institutional Review Board (IRB No. E-2010013) of Pusan National University Hospital Clinical Trial Center. The drug is not covered by healthcare insurance in Korea; thus, the cost is high. The consent document stated clearly that the off-label use of rFVIIa was very expensive and that complications such as coronary artery disease and ischemic stroke might develop upon treatment. rFVIIa was given to patients who did not recover from their coagulation disorders after standard fluid and transfusion treatments, and whose vital signs 
became exacerbated or were not expected to improve. In this study, we gave $250 \mathrm{KIU}(5 \mathrm{mg})$ as an intravenous bolus, thus without considering patient weight [5].

\section{3) Statistical analysis}

Nominal variables are presented as frequencies with percentages. Continuous variables are presented as means with standard deviations when the data were normally distributed. For variables that did not follow normal distributions, the data are presented as medians with interquartile ranges. The Mann-Whitney U-test was used to compare between group differences and a result was considered significant only when the P-value was less than 0.05. We used MedCalc Statistical Software version 13.1 (MedCalc Software, Ostend, Belgium) for all statistical analyses.

\section{Results}

We treated 66 patients with PPH during the study period; average age was $32.2 \pm 5.65$ years. There were 15 patients with SOFA scores $\geq 8$. Eight of these 15 patients received injections of rFVIIa whereas seven patients did not (Figure 1). Of the patients treated with rFVIIa, six were primigravida and two multigravida. Of the patients not treated with rFVIIa, four were primigravida and three multigravida. The mean age of the patients included in the analysis was $31.7 \pm 7.5$ years.

The initial median SOFA score was 10 (interquartile range [IQR], 9.25 to 11.75 ) in the rFVIIa-use group and 9 (IQR, 8.0 to 10.0) in the non-use group. After 24 hours, the eight patients who received rFVIIa had a median SOFA score of 4 (IQR, 2.25 to 4.75) whereas patients who did not receive rFVIIa had a median score of 5 (IQR, 3.0 to 7.0 ) (Figure 2). Thus, the median SOFA score did not differ significantly between the two groups. However, the median $\triangle$ SOFA value was 6.0 (IQR, 5.3 to 8.5) for the eight patients who recieved rFVIIa and 4.0 (3.0 to 5.0) for the seven patients who did not receive such treatment $(\mathrm{P}=0.016)$ (Table 1, Figure 3).
On comparing the two groups, no significant differences in terms of the transfusion levels of red blood cell concentrate, fresh frozen plasma, or platelet concentrate were evident. Also, there was no significant difference in the duration of either intensive care unit (ICU) stay or hospital stay (Table 1).

\section{Discussion}

Although the incidence of PPH has decreased over the

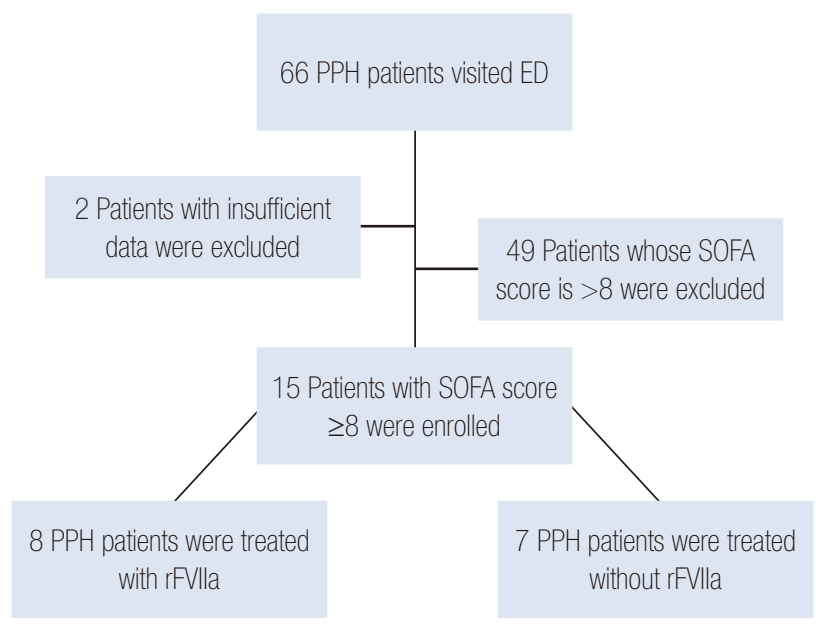

Figure 1. Flow diagram of patients included in this study. PPH: postpartum hemorrhage; ED: emergency department; SOFA: Sequential Organ Failure Assessment; rFVIla: recombinant activated factor VII.

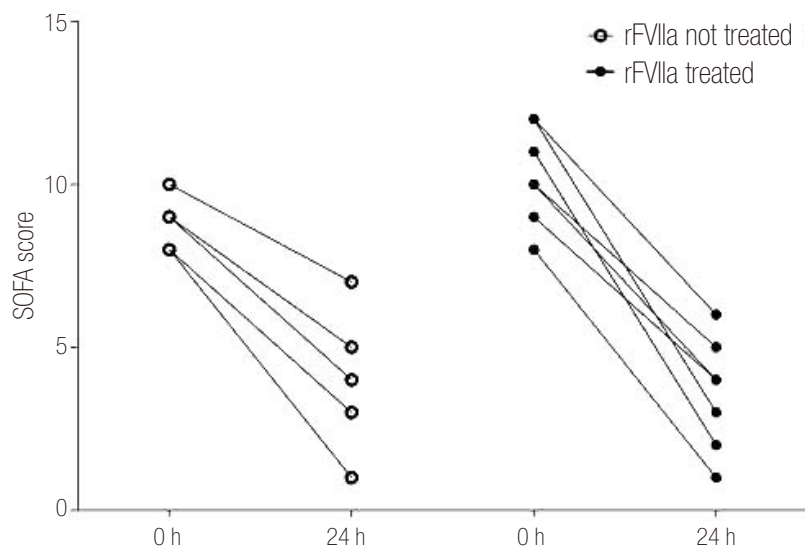

Figure 2. Sequential Organ Failure Assessment (SOFA) scores at 0 and 24 hours for the two groups. rFVIla: recombinant activated factor VII. 
Table 1. Comparison of general characteristics and clinical data between treated with rFVIla group and not treated with rFVIla group

\begin{tabular}{|c|c|c|c|}
\hline Variable & Treated with rFVIla $(n=8)$ & Not treated with $\mathrm{rFVIIa}(\mathrm{n}=7)$ & P-value \\
\hline Age (yr) & $33.0 \pm 7.6$ & $30.1 \pm 7.7$ & 0.505 \\
\hline \multicolumn{4}{|l|}{ Conditions at presentation } \\
\hline Systolic blood pressure (mmHg) & $76.0(50.0-107.5)$ & $60.0(12.5-75.0)$ & 0.416 \\
\hline Heart rate (/min) & $116.5(105.0-133.5)$ & $118.0(111.3-129.0)$ & 0.908 \\
\hline Hemoglobin (g/dl) & $9.7(8.1-11.6)$ & $6.6(5.5-8.5)$ & 0.130 \\
\hline Prothrombin time (s) & $17.0(13.6-19.6)$ & $32.8(18.0-100.0)$ & 0.082 \\
\hline International normalization ratio & $1.53(1.23-1.72)$ & $2.99(1.60-10.00)$ & 0.082 \\
\hline aPTT (s) & $53.3(45.2-65.1)$ & $131.2(55.5-180.0)$ & 0.223 \\
\hline \multicolumn{4}{|l|}{ Transfusion during the first 48 hours } \\
\hline Red blood cell concentrate (unit) & $14.5(10.8-29.5)$ & $17.0(6.0-19.0)$ & 0.613 \\
\hline Fresh frozen plasma (unit) & $13.0(4.0-26.5)$ & $15.0(9.0-19.0)$ & 0.955 \\
\hline Platelet concentrate (unit) & $24.0(8.0-35.0)$ & $16.0(8.0-32.0)$ & 0.536 \\
\hline SOFA at presentation & $10.0(9.3-11.8)$ & $9.0(8.0-10.0)$ & 0.064 \\
\hline SOFA at 24 hours & $4.0(2.3-4.8)$ & $5.0(3.0-7.0)$ & 0.318 \\
\hline$\triangle S O F A$ & $6.0(5.3-8.5)$ & $4.0(3.0-5.0)$ & $0.016^{a}$ \\
\hline ICU stay (h) & $64.1(55.3-108.0)$ & $51.0(29.8-81.0)$ & 0.203 \\
\hline Hospital day & $15.5(10.5-21.5)$ & $12.0(8.0-23.0)$ & 0.523 \\
\hline
\end{tabular}

Values are presented as mean \pm standard deviation or median (interquartile range).

rFVlla: recombinant activated factor VII; aPPT: activated partial thromboplastin time; SOFA: Sequential Organ Failure Assessment; $\triangle$ SOFA: differences between SOFA scores at 24 hours and SOFA scores at presentation; ICU: intensive care unit.

${ }^{a}$ Statistically significant.

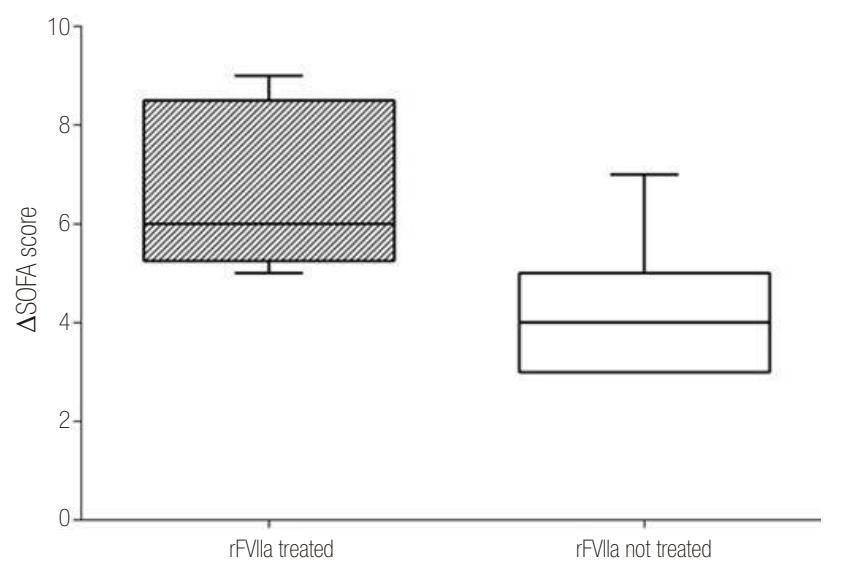

Figure 3. Differences between SOFA scores at 24 hours and SOFA scores at presentation $(\triangle \mathrm{SOFA})$ for the two groups. SOFA: Sequential Organ Failure Assessment; rFVIla: recombinant activated factor VII.

past 40 years, morbidity and mortality associated with obstetric hemorrhage remain high; this is a potentially life-threatening obstetric complication worldwide [1,2]. The standard PPH management consists of initial nonin- vasive nonsurgical interventions. If necessary, invasive and surgical treatments follow. These include injection of crystalloid and blood components to restore blood volume, uterine massage, uterotonic medications such as oxytocin, uterine compression suturing, vessel ligation, and embolization of the uterine or iliac arteries [4]. In emergency departments, replacing blood volume via massive and aggressive transfusions of crystalloid may trigger serious complications such as coagulopathy, hypothermia, or metabolic acidosis. Also, even the optimal treatments used to arrest obstetric hemorrhage and prevent the need for emergency hysterectomy are only about $50 \%$ effective [6].

In recent years, rFVIIa has emerged as a novel therapy for the treatment of major PPH unresponsive to conventional therapies, not only in emergency departments but also in the operative field (Figure 4). Numerous reports of successful off-label use of rFVIIa for the management of major obstetric hemorrhage have appeared. In 


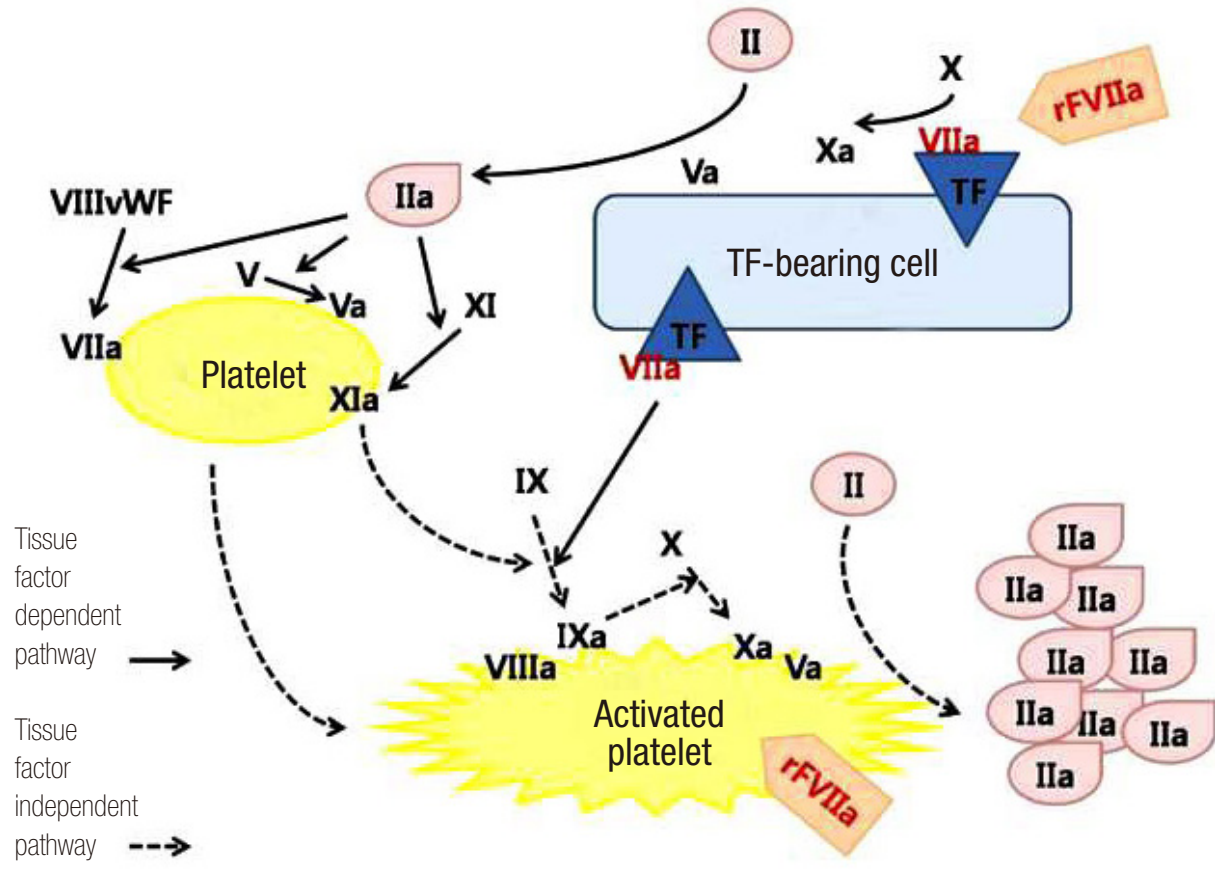

Figure 4. Clotting mechanism and action of rFVIla on injured tissue. vWF: von Willebrand factor; TF: tissue factor; rFVIla: recombinant activated factor VII.
2001, the first successful case was reported. That patient received rFVIIa to treat $\mathrm{PPH}$ associated with disseminated intravascular coagulation, liver failure, and renal dysfunction [7]. Between 2000 and 2004, a large series of 113 cases were successfully treated in nine European countries; $80 \%$ of cases exhibited marked improvement with very few side-effects [8].

In 2006, the European Society of Emergency Medicine, the European Society for Trauma and Emergency Surgery, the European Society of Intensive Care Medicine, the European Society of Anesthesiology, and the European Hematology Association together published revised guidelines for rFVIIa use in the treatment of uncontrolled $\mathrm{PPH}$ [9]. Also, an American review group suggested that rFVIIa used to treat PPH was appropriate after failure of clotting factor replacement [10].

In the present study, the amounts of transfused red blood cell concentrate and fresh frozen plasma decreased in most patients following administration of rFVIIa, but the total amounts did not fall significantly. However, we found that the use of rFVIIa to treat severe PPH enhanced SOFA score reduction. Thus, the magnitude of severe PPH can be diminished by rFVIIa use.
The $\triangle$ SOFA values correlated with mortality in several studies. Jones et al. [11] analyzed 248 patients with severe sepsis and septic shock and found that initial SOFA score and the $\triangle$ SOFA (initial-72 hours) value correlated strongly with mortality. Any increase in the $\triangle \mathrm{SOFA}$ value was associated with a $35 \%$ in-hospital mortality rate, whereas any decrease in the $\triangle \mathrm{SOFA}$ value was associated with $10 \%$ mortality. Ferreira et al. [12] studied 352 patients admitted to a surgical ICU and demonstrated that the mean SOFA score and $\triangle$ SOFA value correlated strongly with mortality. Thus, we used the $\triangle$ SOFA value to assess the response to treatment, as have other studies [13].

For decades, no clear guidelines for rFVIIa use in situations of massive bleeding have been available. Thus, the doses of rFVIIa used vary among clinicians, ranging from 15 to $120 \mu \mathrm{g} / \mathrm{kg}$; a single dose usually affords a good result [14]. One report found that a low dose of $\mathrm{rF}$ VIIa (i.e., $<20 \mu \mathrm{g} / \mathrm{kg}$ ) successfully arrested small arterial bleeding [14].

In our present retrospective cohort study, the amount of rFVIIa given was constant (i.e., $250 \mathrm{KIU}$ ), thus not based on individual weight, and we did not give repeat injections. Gathering of personal information such as 
weight in emergency situations is not easy, and the cost of the agent cannot be excluded as a valid consideration. Had we based the rFVIIa levels on individual weight, it would have been possible to evaluate effectiveness more accurately.

The use of rFVIIa is often controversial among clinicians due to the associated complications, especially thrombotic events. In this study, no such complications were encountered. The U.S. Food and Drug Administration warns clinicians of the increased risk of thromboembolic events after rFVIIa administration, particularly in those who have or may have preexisting risk factors. Thus, in practice, clinicians often hesitate to use rFVIIa even in situations of life-threatening obstetric hemorrhage. However, the incidence of adverse events after rFVIIa infusion was only 25 in 100,000 [15]. Bomken et al. [16] reported no thromboembolic event in 15 patients given rFVIIa injections despite the relative prothrombotic states of their pregnancies. In general, many thromboembolic adverse effects developing after rFVIIa injection occurred in patients with preexisting risk factors [17]. Thus, the use of rFVIIa to treat severe PPH is essentially unassociated with thromboembolic events. Clinicians should not be influenced by the controversy; rFVIIa may be considered a safe hemostatic agent for control of major obstetric bleeding.

Our study had several limitations. First, we did not measure fibrinogen levels because such measurement is not routine when patients arrive in the emergency department; the associated laboratory time is long. Thus, we lacked such data. Therefore, the initial and final levels of fibrinogen and platelets did not appear on our data sheets. Second, our patient numbers were small because assessment of rFVIIa off-label use remains incomplete, and the treatment is expensive. Third, we retrospectively reviewed patient data. The absence of randomized controlled studies on rFVIIa use in PPH renders the evidence for such use weak and the data controversial. Several worldwide randomized controlled trials are required to establish clear guidelines for rFVIIa use in cases of major obstetric hemorrhage, and to put the controversy to rest.
In conclusion, we found that the use of rFVIIa to treat severe PPH reduced SOFA score. rFVIIa could be employed as an adjunctive therapy in such cases. Further study is needed, given our small sample size. We view the present work as a pilot study.

\section{Acknowledgments}

This work was supported by a 2-year Research Grant from Pusan National University.

\section{ORCID}

$\begin{array}{ll}\text { Soon Chang Park } & \text { http://orcid.org/0000-0003-2271-0946 } \\ \text { Seok Ran Yeom } & \text { http://orcid.org/0000-0002-1000-9637 }\end{array}$

\section{References}

1. Say L, Chou D, Gemmill A, Tunçalp Ö, Moller AB, Daniels J, et al. Global causes of maternal death: a WHO systematic analysis. Lancet Glob Health 2014;2:e323-33.

2. Statistics Korea. Korean statistical information service, infant, maternal and perinatal mortality statistics 2014. Daejeon: Statistics Korea; 2015.

3. Henry A, Birch MR, Sullivan EA, Katz S, Wang YA. Primary postpartum haemorrhage in an Australian tertiary hospital: a case-control study. Aust N Z J Obstet Gynaecol 2005;45:233-6.

4. World Health Organization. WHO recommendations for the prevention and treatment of postpartum haemorrhage: evidence base [Internet]. Geneva: World Health Organization; 2012 [cited 2017 Oct 16]. Available from: www.who.int/reproductivehealth/publications/maternal_perinatal_health/9789241548502/en.

5. Welsh A, McLintock C, Gatt S, Somerset D, Popham $\mathrm{P}$, Ogle R. Guidelines for the use of recombinant activated factor VII in massive obstetric haemorrhage. Aust N Z J Obstet Gynaecol 2008;48:12-6.

6. Yamamoto H, Sagae S, Nishikawa S, Kudo R. Emer- 
gency postpartum hysterectomy in obstetric practice. J Obstet Gynaecol Res 2000;26:341-5.

7. Moscardo F, Perez F, de la Rubia J, Balerdi B, Lorenzo JI, Senent ML, et al. Successful treatment of severe intra-abdominal bleeding associated with disseminated intravascular coagulation using recombinant activated factor VII. Br J Haematol 2001;114:174-6.

8. Alfirevic Z, Elbourne D, Pavord S, Bolte A, Van Geijn H, Mercier F, et al. Use of recombinant activated factor VII in primary postpartum hemorrhage: the Northern European registry 2000-2004. Obstet Gynecol 2007;110:1270-8.

9. Vincent JL, Rossaint R, Riou B, Ozier Y, Zideman D, Spahn DR. Recommendations on the use of recombinant activated factor VII as an adjunctive treatment for massive bleeding: a European perspective. Crit Care 2006;10:R120.

10. Shander A, Goodnough LT, Ratko T, Matuszewski KA, Cohn PS, Diringer M, et al. Consensus recommendations for the off-label use of recombinant human factor VIIa (NovoSeven ${ }^{\circledR}$ ) therapy. P T 2005;30:644-58.

11. Jones AE, Trzeciak S, Kline JA. The Sequential Organ Failure Assessment score for predicting outcome in patients with severe sepsis and evidence of hypoperfusion at the time of emergency department presentation. Crit Care Med 2009;37:1649-54.
12. Ferreira FL, Bota DP, Bross A, Melot C, Vincent JL. Serial evaluation of the SOFA score to predict outcome in critically ill patients. JAMA 2001;286:17548.

13. de Azevedo JR, Torres OJ, Beraldi RA, Ribas CA, Malafaia O. Prognostic evaluation of severe sepsis and septic shock: procalcitonin clearance vs $\Delta$ Sequential Organ Failure Assessment. J Crit Care 2015;30:219.e9-12.

14. Ogawa M, Akahira S, Takahashi S, Shimoda Y, Sato M, Sato A, et al. Low-dose recombinant activated factor VII temporally stopped bleeding from small artery in severe postpartum hemorrhage: a case report. Blood Coagul Fibrinolysis 2013;24:344-6.

15. Aledort LM. Comparative thrombotic event incidence after infusion of recombinant factor VIIa versus factor VIII inhibitor bypass activity. J Thromb Haemost 2004;2:1700-8.

16. Bomken C, Mathai S, Biss T, Loughney A, Hanley J. Recombinant activated factor VII (rFVIIa) in the management of major obstetric haemorrhage: a case series and a proposed guideline for use. Obstet Gynecol Int 2009;2009:364843.

17. Biss TT, Hanley JP. Recombinant activated factor VII (rFVIIa/NovoSeven) in intractable haemorrhage: use of a clinical scoring system to predict outcome. Vox Sang 2006;90:45-52. 